Narrative Review

\title{
Digital Subtraction Angiography Use During Epidural Steroid Injections Does Not Reliably Distinguish Artery from Vein
}

Ameet S. Nagpal, MD'1, George C. Chang Chien, DO², Jonathan A. Benfield, DO, Kenneth D. Candido, MD ${ }^{4}$, Maunak V. Rana, MD ${ }^{4}$, and Maxim S. Eckmann, MD ${ }^{1}$

From: ${ }^{1}$ Department of Anesthesiology, University of Texas Health Science Centerat

San Antonio; ${ }^{2}$ Department

of Anesthesiology \& Pain Management, Cleveland Clinic

Foundation; ${ }^{3}$ Department of Physical Medicine \&

Rehabilitation, University of

Texas Health Science Center

San Antonio; ${ }^{4}$ Department of Anesthesiology, Advocate Illinois

Masonic Medical Center

Address Correspondence: Jonathan A. Benfield, DO Department of Physical Medicine \& Rehabilitation University of Texas Health Science Center at San Antonio 7703 Floyd Curl Dr. Mail Code 7798 San Antonio, Texas E-mail:

benfield@uthscsa.edu

Disclaimer: There was no external funding in the preparation of this manuscript. Conflict of interest: Each author certifies that he or she, or a member of his or her immediate

family, has no commercial association (i.e., consultancies, stock ownership, equity interest, patent/licensing arrangements, etc.) that might pose a conflict of interest in connection with the submitted manuscript.

Manuscript received: 08-05-2015 Revised manuscript received: 10-13-2015

Accepted for publication: 10-29-2015

Free full manuscript: www.painphysicianjournal.com
Background: Epidural steroid injections (ESIs) are among the most common procedures performed in an interventional pain management practice. It is well known that tragic complications may arise from ESIs, most commonly those performed using a transforaminal approach. Digital subtraction angiography (DSA) has been hailed as a fluoroscopic technique that can be used to detect arterial placement of the injection needle, and therefore as a safety measure that can decrease the incidence of catastrophic sequelae of these procedures.

Objective: The objective of this article was to review existing scientific pain literature to determine if DSA can distinguish arterial vs. venous uptake.

Study Design: Narrative review.

Methods: The current narrative review of DSA in interventional spine was completed with a PUBMED search using the key words: digital subtraction angiography, epidural, fluoroscopy, intravascular injection, paraplegia, and quadriplegia in accordance with Preferred Reporting Items for Systematic Reviews and Meta- Analyses (PRISMA) guidelines.

Results: After identification of duplicate articles, 383 articles were screened by title, abstract, and/or full article review. Ten of these articles were deemed appropriate, after applying inclusion and exclusion criteria, as they specifically looked at the use of digital subtraction angiography in interventional spine epidural injections. This included 4 case reports, 3 prospective studies, one retrospective analysis, one prospective cohort study, and one meta-analysis.

All of the available studies claiming that DSA was capable of detecting vascular spread are likely accurate, but no significant detection of specifically arterial spread has been reported. The known catastrophic complications related to ESIs are purported to be due to arterial injection of insoluble steroids or local anesthetic and detection of arterial spread of contrast during fluoroscopy would be of obvious benefit to the interventionalist.

Limitations: Small study size, non-randomized studies between DSA and real time fluoroscopy.

Conclusion: Existing studies do not support that DSA can predict arterial spread. In fact, DSA exposes the practitioner and the patient to higher levels of radiation without objective evidence of any safety parameters.

Key words: Digital subtraction angiography, real-time fluoroscopy, transforaminal epidural injection, particulate steroids, cervical radicular artery, lumbar radicular artery, spinal cord injury

Pain Physician 2016; 19:255-266 
pidural steroid injections (ESIs) are common and effective therapeutic interventions within the multimodal management of spinal pain conditions arising from nerve root irritation due to inflammatory mediators accessing nociceptors. Neuraxial delivery of corticosteroids and/or local anesthetic has an excellent safety profile with a favorable risk-benefit ratio. Serious complications from neuraxial anesthetic blocks are rare (1) and are estimated at approximately up to 5.0 per 100,000 cases in the setting of chronic pain management (2). Proactive prevention of rare catastrophes such as irreversible quadriplegia or paraplegia, stroke, blindness, cerebellar herniation, transverse myelitis, fungal infections, arachnoiditis, aseptic meningitis, and death have motivated the physician-scientist to re-examine the administration of steroids into the epidural space and attempt to identify the etiology of the above mentioned catastrophes (36). Digital subtraction angiography (DSA) has been one of many measures promoted as a radiologic adjunct to minimize complications from interventional neuraxial procedures where it is imperative to identify vascular, especially arterial, access during the injection.

DSA software is employed to obtain images of vascular structures in the body and is used commonly by specialties other than interventional pain physicians. Its primary use is the evaluation of large extra and intracranial, cardiac, pulmonary, and renal vessels for occlusions, hypertension, and pre-operative evaluation for vascular lesions (7). DSA is theoretically considered to be useful in interventional spine fluoroscopy procedures because it is highly effective in contrasting vascular structures with their surrounding bone and soft tissue. DSA is commonly used as an adjunctive safety measure in high-risk procedures such as cervical transforaminal epidural steroid injection (TFESI), cervical and lumbar interlaminar epidural steroid injection (ILESI), caudal epidural steroid injection (ESI), discography, and adhesiolysis (8-10). Its name describes its function: DSA digitally "subtracts" a baseline radiograph from serial images which display changes in the contrast appearance over time. When contrast medium is injected, $x$-ray detection of the contrast medium produces 1 to 30 exposures per second both pre- and post-contrast media injection. The digital image software process subtracts the pre-contrast images from the post-contrast injection. The data is then saved and viewed as a short video clip for detection of vascular structures and potential vascular uptake of contrast media injectate; a so-called "cine-angiography" type of process. DSA has emerged as an additional supportive measure in identifying vascular trespass and has demonstrated greater accuracy in detecting intravascular injections compared to aspiration and live fluoroscopy (9-11).

Although numerous authors suggest that DSA may be superior to live fluoroscopy in reducing neurologic injury associated with interventional pain medicine procedures, the data supporting this belief is not confirmatory. Recently, studies have questioned DSA's accuracy in identifying arterial versus venous vasculature uptake (12) and its superiority to live fluoroscopy $(11,13-15)$.

The present narrative review evaluates DSA as a modality which has been proposed to avoid arterial related adverse events while performing cervical, thoracic or lumbar ESI from TFESI, ILESI (including parasagittal and paramedian approaches), and caudal approaches. The necessity of maintaining vascular integrity during interventional spine injections is to prevent intraarterial uptake of injectate into the central nervous system and ensuring local deposition of medication to the nociceptive target, thus decreasing the possibility of a false negative block, as well as potentially limiting or detecting ischemic infarction, respiratory depression, and seizures due to arterial uptake and potential delivery to the spinal cord or brain. Although of significant concern, extra-spinal and venous deposition of medication are most likely to be inconsequential. This narrative review focuses on the role of DSA to detect arterial vs. venous vascular uptake.

\section{Methods}

\section{Literature Search Strategy}

The current narrative review of DSA in interventional spine completed a PUBMED search using the key words: digital subtraction angiography, epidural, fluoroscopy, intravascular injection, paraplegia, and quadriplegia in accordance Preferred Reporting Items for Systematic Reviews and Meta- Analyses (PRISMA) flow sheet (Fig. 1), checklist, and Explanation and Elaboration document which can be found at www. prisma-statement.org/statement.htm. Fig. 1 displays the PRISMA flowsheet for the literature search strategy which represents the initial search using the key words "digital subtraction angiography" which resulted in 11,896 articles. Using the following key words and combining those key words (digital subtraction angiography, fluoroscopy, epidural, intravascular injection paraplegia, quadriplegia) resulted in 395 articles. Ten articles were selected using inclusion criteria. Those 
Records identified through database searching (PubMed) using keywords "digital subtraction angiography". $(\mathrm{n}=12,000)$
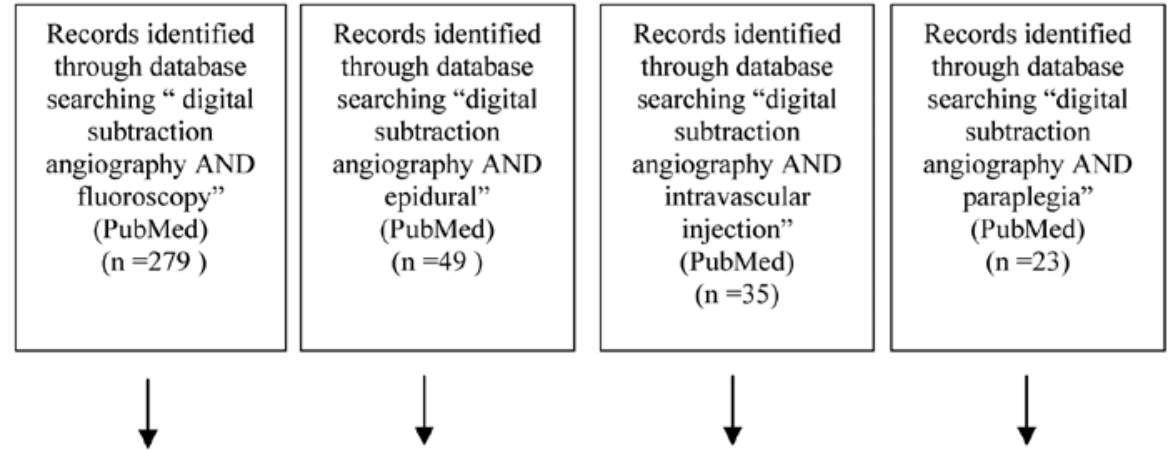

Records identified through database searching "digital digital subtraction angiography AND quadriplegia" (PubMed) $(\mathrm{n}=9)$

Records identified through database searching using a combination of keywords above $(279+49+35+23+9=$ 395) (PubMed) ( $n=395)$

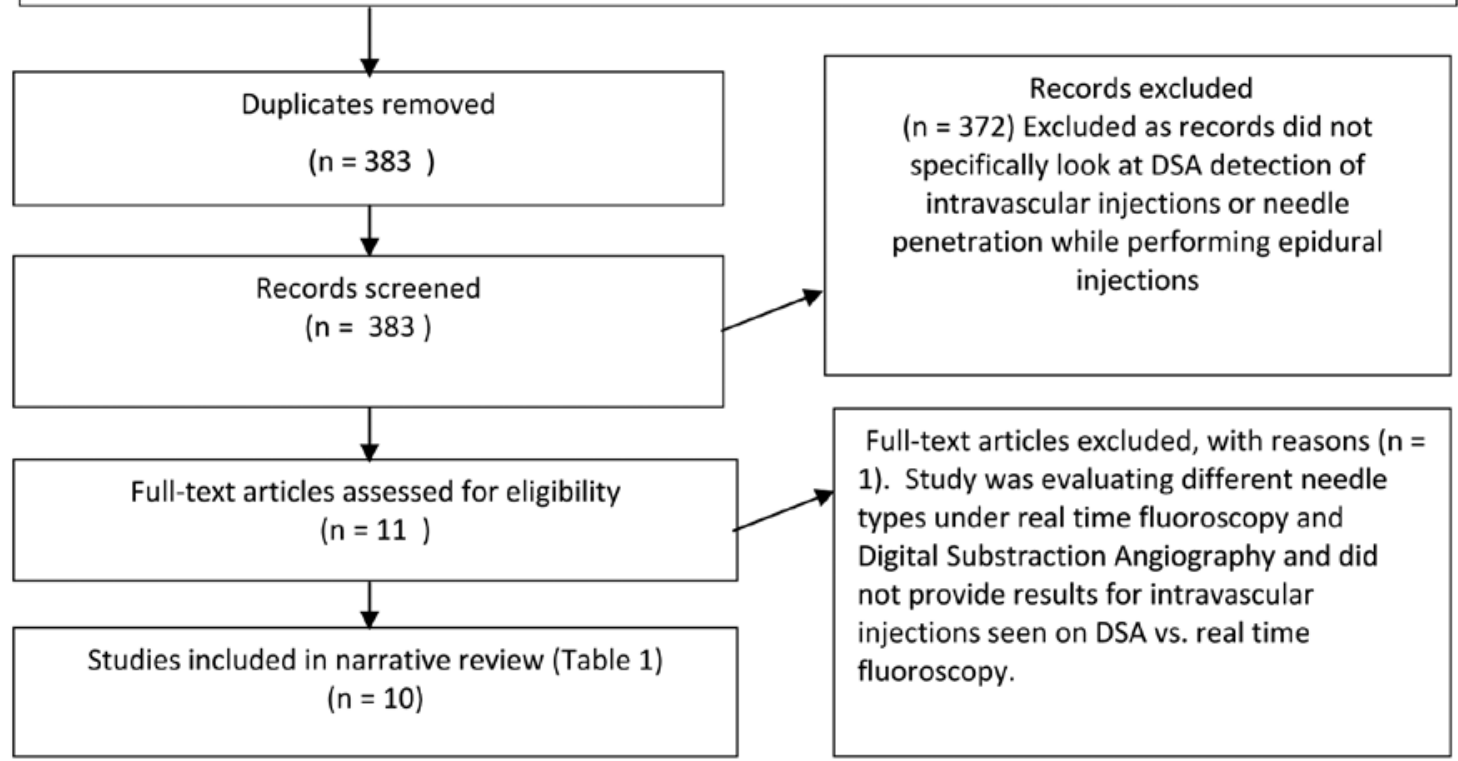

Fig. 1. PRISMA flowsheet.

10 articles included one meta-analysis, 3 prospective studies, one prospective cohort study, one retrospective analysis, and 4 case reports.

\section{Inclusion \& Exclusion Criteria}

Ten of these articles were deemed appropriate as they specifically looked at the use of digital subtraction angiography in interventional spine epidural injections
(Table 1). Three hundred seventy-three articles were excluded as records did not specifically look or report on DSA detection of intravascular injections or needle penetration while performing epidural injections. Limiting bias for reviewed articles included selective reporting and publication bias as most studies reviewed did not differentiate between arterial and vascular uptake and supported the use of DSA in epidural injections (i.e., 
Table 1. DSA studies comparing fluoroscopy, DSA, and additional safety measures.

\begin{tabular}{|c|c|c|c|c|}
\hline Study & Intervention & Results & Comments & $\begin{array}{l}\text { Level \& } \\
\text { Grades }\end{array}$ \\
\hline Baker et al (16) & $\begin{array}{l}\text { One Cervical TFESI } \\
\text { Case Report }\end{array}$ & $\begin{array}{l}\text { Vascular uptake on fluoroscopy and } \\
\text { DSA }\end{array}$ & $\begin{array}{l}\text { Was DSA necessary to confirm when } \\
\text { fluoroscopy identified vascular uptake? }\end{array}$ & $\begin{array}{l}\mathrm{III} \\
\mathrm{C}\end{array}$ \\
\hline $\begin{array}{l}\text { Verrill et al (17) } \\
\text { Landers (18) }\end{array}$ & $\begin{array}{l}\text { One Cervical TFESI } \\
\text { Case Report }\end{array}$ & DSA detected vascular etiology & $\begin{array}{l}\text { Incorrect needle placement detected on } \\
\text { fluoroscopy according to ISIS guidelines. } \\
\text { Inappropriate justification for DSA. }\end{array}$ & $\begin{array}{l}\text { III } \\
\text { C }\end{array}$ \\
\hline Jasper (8) & $\begin{array}{l}\text { One Atlanto occipital } \\
\text { Injection, CESI, and } \\
\text { Cervical TFESI } \\
\text { Case Report }\end{array}$ & DSA demonstrated vascular etiology & $\begin{array}{l}\text { Only DSA used. Unable to compare DSA and } \\
\text { fluoroscopy directly. }\end{array}$ & $\begin{array}{l}\text { III } \\
\text { C }\end{array}$ \\
\hline $\begin{array}{l}\text { Chang Chien et } \\
\text { al (4) }\end{array}$ & $\begin{array}{l}\text { One Lumbar TFESI } \\
\text { Case Report }\end{array}$ & $\begin{array}{l}\text { Patient with thoracic spinal cord } \\
\text { infarct. Initially T7-8 with later } \\
\text { imaging showing T6-10. }\end{array}$ & DSA did not detect vascular uptake. & $\begin{array}{l}\text { III } \\
\text { C }\end{array}$ \\
\hline McLean et al (11) & $\begin{array}{l}177 \text { cervical TFESI } \\
\text { Retrospective } \\
\text { Analysis }\end{array}$ & $\begin{array}{l}18 \% \text { detected via fluoroscopy. } \\
32.8 \% \text { detected via DSA. }\end{array}$ & $\begin{array}{l}\text { All of the vascular angiograms identified by } \\
\text { both live fluoroscopy and by DSA lumbosacral } \\
\text { were noted to venous in nature. Although } \\
\text { DSA discovered more venous vascular uptake } \\
\text { it did not discover any more arterial uptake } \\
\text { than fluoroscopy. }\end{array}$ & $\begin{array}{c}\text { III } \\
\text { B }\end{array}$ \\
\hline Lee et al (9) & $\begin{array}{l}87 \text { lumbar/sacral } \\
\text { TFESI } \\
\text { Prospective Study }\end{array}$ & $\begin{array}{l}12 \text { cases with vascular uptake } \\
\text { detected by fluoroscopy and DSA, } 8 \\
\text { by DSA only }\end{array}$ & $\begin{array}{l}\text { Authors did not distinguish arterial or venous } \\
\text { uptake. }\end{array}$ & $\begin{array}{l}\text { IIA } \\
\text { B }\end{array}$ \\
\hline El Abd et al (10) & $\begin{array}{l}222 \text { cervical, lumbar, } \\
\text { and sacral TFESI } \\
\text { Prospective Cohort } \\
\text { Study }\end{array}$ & $\begin{array}{l}\text { Cervical }=18.47 \% \\
\text { Lumbar }=50.9 \% \\
\text { Sacral }=30.36 \% \\
\text { Identified vascular uptake by } \\
\text { additional safety measures with DSA } \\
\text { detecting } 5 \text { additional cases. }\end{array}$ & $\begin{array}{l}\text { All } 5 \text { additional cases detected by DSA were } \\
\text { during sacral TFESI and venous in nature. }\end{array}$ & $\begin{array}{l}\text { IIA } \\
\text { B }\end{array}$ \\
\hline Kim et al (15) & $\begin{array}{l}732 \text { lumbar TFESI } \\
\text { Prospective Study }\end{array}$ & $\begin{array}{l}\text { Fluoroscopy }=8.1 \% \\
\text { DSA }=10.5 \% \text { vascular uptake, } P= \\
0.13\end{array}$ & $\begin{array}{l}\text { No statistical difference between fluoroscopy } \\
\text { or DSA detected vascular uptake. }\end{array}$ & $\begin{array}{l}\text { IIA } \\
\text { B }\end{array}$ \\
\hline Hong et al (21) & $\begin{array}{l}249 \text { L1-S1 TFESI } \\
\text { Prospective Study }\end{array}$ & $\begin{array}{l}\text { 31/249 with vascular uptake. } \\
\text { 9/31 detected by DSA alone. }\end{array}$ & $\begin{array}{l}4 \text { case of the DSA alone had partial vascular } \\
\text { uptake on fluoroscopy. Authors did not } \\
\text { distinguish between arterial and venous } \\
\text { uptake. }\end{array}$ & $\begin{array}{l}\text { IIA } \\
\text { B }\end{array}$ \\
\hline $\begin{array}{l}\text { Visnjevec et al } \\
(22)\end{array}$ & $\begin{array}{l}1290 \text { TFESI } \\
\text { Meta-analysis of four } \\
\text { prospective studies }\end{array}$ & $\begin{array}{l}188 \text { events detected by DSA and/or } \\
\text { real-time fluoroscopy. } 40 \text { were DSA } \\
\text { alone. }\end{array}$ & $\begin{array}{l}\text { Only used TFESI so unable to extrapolate to } \\
\text { ILESI, no randomized studies, } 40 \text { false positive } \\
\text { and } 0 \text { false negative TFESI. }\end{array}$ & $\begin{array}{l}\text { IIB } \\
\mathrm{B}\end{array}$ \\
\hline
\end{tabular}

favoring a new intervention as beneficial). There were also a limited number or studies in general and none of those studies were randomized control studies.

\section{Results}

\section{Case Reports and Case Series}

The case illustrated in Fig. 2 involved a 40-year old woman with a history of cervical radicular pain who was scheduled to undergo a left C6-7 TFESI. Initial placement of the needle was done under C-arm fluoroscopic guidance using anterior-oblique and posterior views. A test dose of $0.5 \mathrm{~mL}$ of contrast medium was injected. Post-injection imaging showed contrast spread extending outside of the intervertebral foramen; therefore the needle was advanced $2 \mathrm{~mm}$ medially. A second test dose performed under fluoroscopy demonstrated contrast spread which was deemed insufficient for the volume of contrast that was injected. Therefore, DSA was used to confirm a suspicion of an intravascular needle placement. DSA imaging demonstrated contrast entering a small transverse vessel leading to the spinal cord. The procedure was aborted and the patient suffered no adverse reactions. In this instance the arterial injec- 


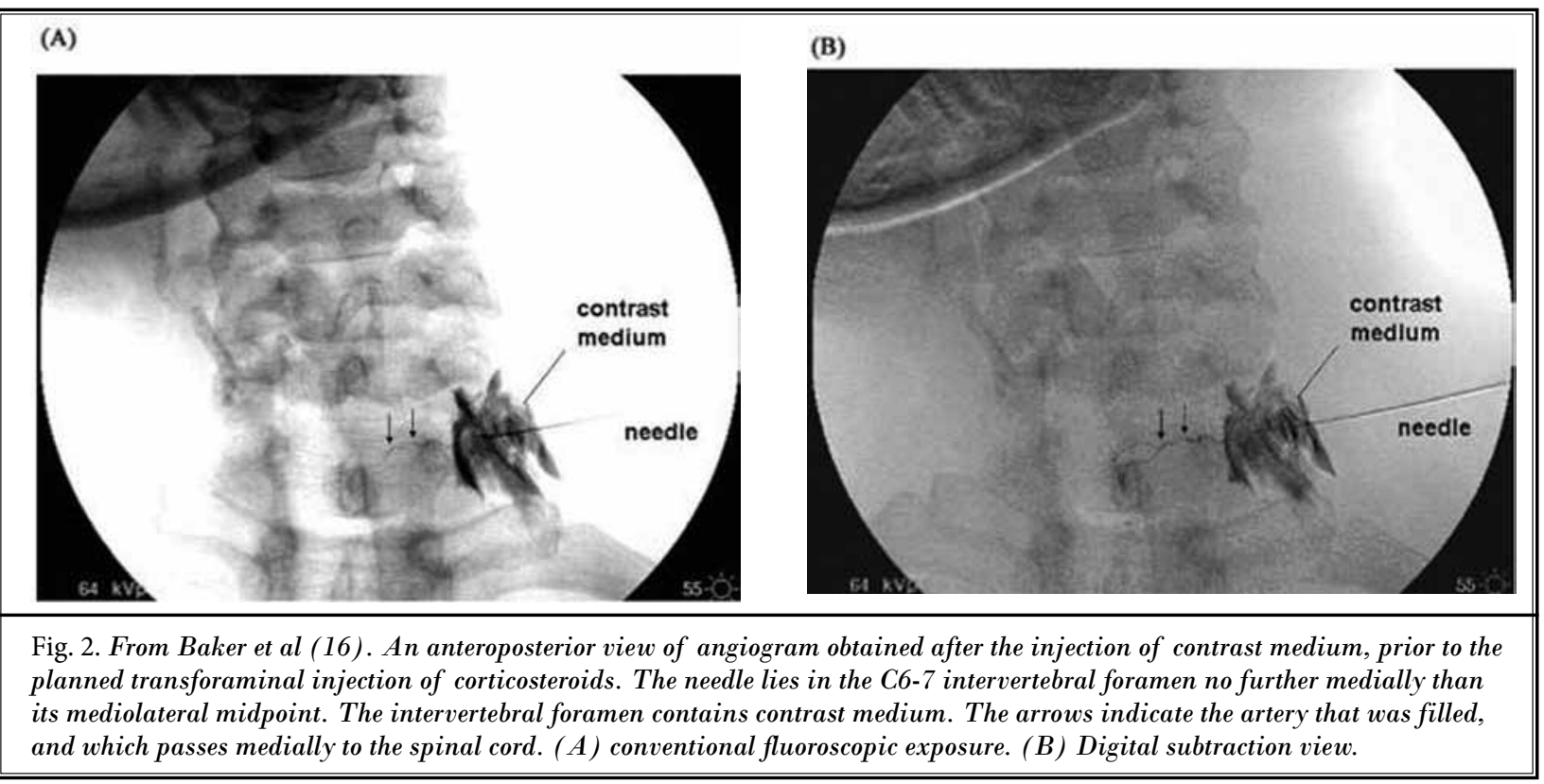

tion was not visualized under fluoroscopy but was seen under DSA. However, the physician was able to identify the lack of contrast and proceeded to verify placement prior to steroid injection with DSA which revealed the arterial placement. Here, an abnormality was initially suspected using traditional methods but was confirmed with DSA. Interestingly, both the images of live fluoroscopy and digital subtraction imaging provided by the authors in their published manuscript clearly demonstrate the transverse vascular flow pattern heading medially towards the center of the spinal column. An obvious conclusion is that DSA was unnecessary as the suspected arterial spread was already visualized under live fluoroscopy (16). DSA increased exposure to ionizing radiation for the patient and the interventionalists in this case, while not providing any additional diagnostic value or changing the outcome of the decisionmaking process necessary to abort the injection.
In the case described by Verrills et al (17) (Fig. 3), a 49 year-old man with 66 radicular pain due to an osteophyte compressing his left $\mathrm{C} 6$ nerve root and spinal cord is reported. He had already had a successful TFESI 8 months previously. During this second TFESI, initial needle placement was confirmed with fluoroscopy on posterior-anterior and oblique views. There was no blood noted on aspiration. A test dose of contrast was injected using DSA. Upon injection, cannulation of a cervical radicular artery occurred and intravascular uptake of contrast was recorded. The images of this procedure display a C6 radicular artery directly feeding into the anterior spinal artery. The procedure was appropriately aborted and the patient sustained no adverse reaction or injury. He later underwent a successful cervical TFESI with no vascular uptake on confirmatory imaging and with significant pain relief. In this case, although placement was initially confirmed using

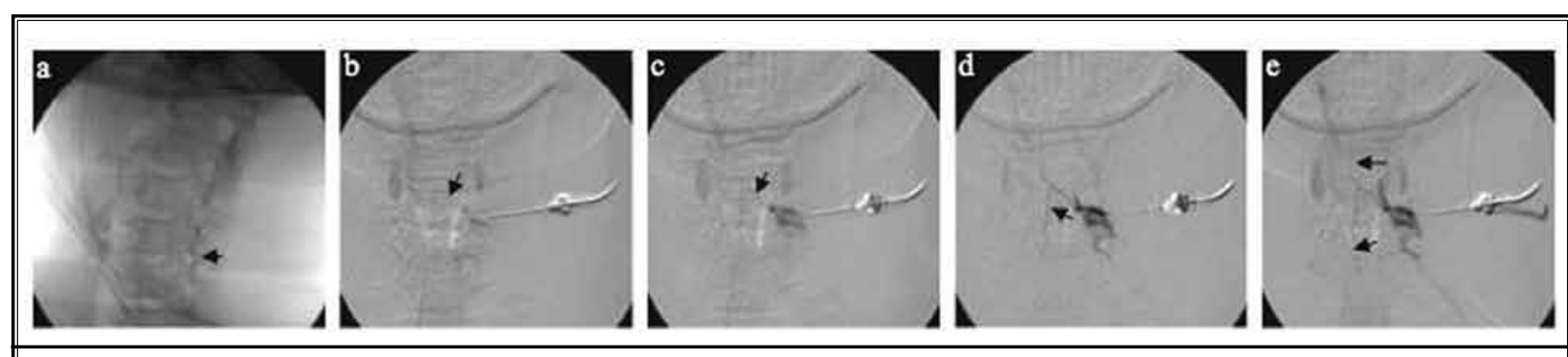

Fig. 3. From Verrills et al (17). Cervical radicular artery ramifying the anterior spinal artery: (a) oblique approach view by $C$-arm conventional fluoroscopy with the needle in the C5/6 foramen; ( $b$ and $c$ ) filling of the cervical radicular artery with contrast under digital subtraction fluoroscopy; and ( $d$ and $e$ ) ramification of the anterior spinal artery by the cervical radicular artery. 


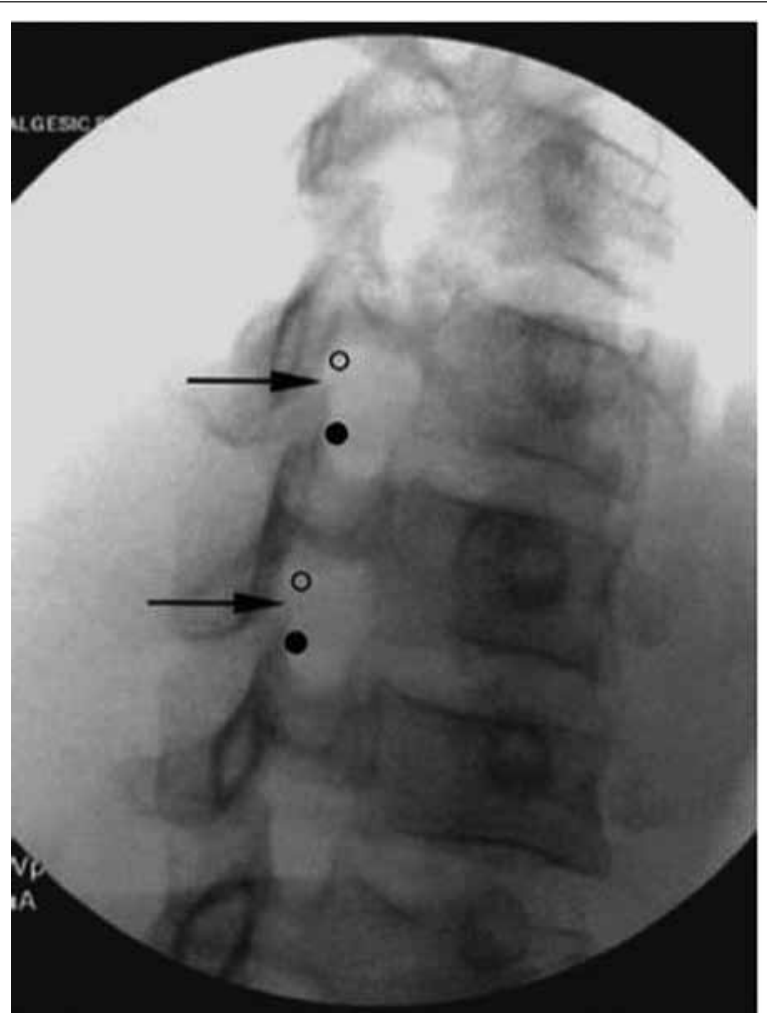

Fig. 4. From Landers (18) "The anterior-oblique, or "foraminal view" of the cervical spine with the C4-5 and C5-6 foramina at their widest diameter both in the cephalocaudal and dorso-ventral dimensions. Arrows indicate the most rostral aspect of the superior articular pillars of $C 5$ and C6. The unfilled circles represent the needle position in the case report. The solid circles represent the needle position as advocated in the ISIS practice guidelines."

fluoroscopy with negative aspirate, the test dose was performed under DSA. At first glance, this case report is promising in regards to DSA detecting arterial uptake. However, Landers's response (18) to this case report in Pain Medicine was published shortly thereafter discussing the merits of this case. Dr. Landers demonstrated (Fig. 4) how the placement of the needle in their case report was not consistent with correct needle placement per International Spine Intervention Society (ISIS) [now known as Spine Intervention Society (SIS)] guidelines. This aberrant needle-artery cannulation could potentially have been avoided with a different needle position. Therefore, correct needle placement potentially obviated the need for DSA if appropriate needle technique and placement had been performed according to ISIS guidelines, which are based upon expert, consensus guidelines. While these guidelines are not necessarily standard of care, the utility of these guidelines as they relate to the performance a procedure with known dangerous complications, such as cervical TFESI, is paramount.

The above 2 cases represent a common conundrum with the use of DSA and its reported ability to distinguish arterial uptake in that it can give a false sense of security to the interventionalist. In this vein, closed claims data (19) provides several interesting facts. There has been an increase in cervical spine procedures, postprocedure injury, and untrained physicians performing these procedures in the last decade. The data also shows that cervical procedures comprise $22 \%$ of all chronic pain management claims (64\% epidural, $11 \%$ stellate ganglion, and $8 \%$ trigger point procedures). Injuries during these procedures included $30 \%$ direct needle trauma to the cord, $9 \%$ intravascular injection, $6 \%$ dural puncture, $5 \%$ spinal block, and 3\% pneumothorax. Eighty-seven percent of the 38 injuries involving the spinal cord resulted in $24 \%$ quadriplegia, $16 \%$ paraplegia, and $8 \%$ hemiplegia. Of note, cases with needle trauma were more likely to be associated with general anesthesia or sedation than without $(71 \%$ vs. $31 \%$ ) (19).

Jasper, in a 2003 case series (8), outlined 3 case reports of TFESI procedures performed using DSA which identified intravascular flow patterns. The first occurred during intra-articular entry of the atlanto-occipital joint wherein the needle penetrated the vertebral artery and the procedure was aborted. The second discussed a caudally directed catheter wherein the first lateral plain film failed to demonstrate significant contrast accumulation. Additional contrast showed a distinct vascular flow with washout upon repeat injection and visualization with a lateral view and posterior-anterior view. Subsequent catheter pullback and reintroduction presented a slightly different intravascular pattern. Final placement produced the desired L5-S1 result. Jasper's final case was a cervical TFESI injection with obvious venous injection within a few seconds of initial contrast injection. After 2 needle adjustments the proper contrast spread was identified. Jasper contended that with single injections the operator may be confronted by a lack of contrast accumulation and may potentially miss the vascular flow patterns seen with DSA. While these cases highlight the rare but known possible side effect of vascular cannulation during TFESI, they were performed using only DSA and not live fluoroscopy. Therefore, it is unknown if these cases of vascular injection would have been identified with live fluoroscopy, 


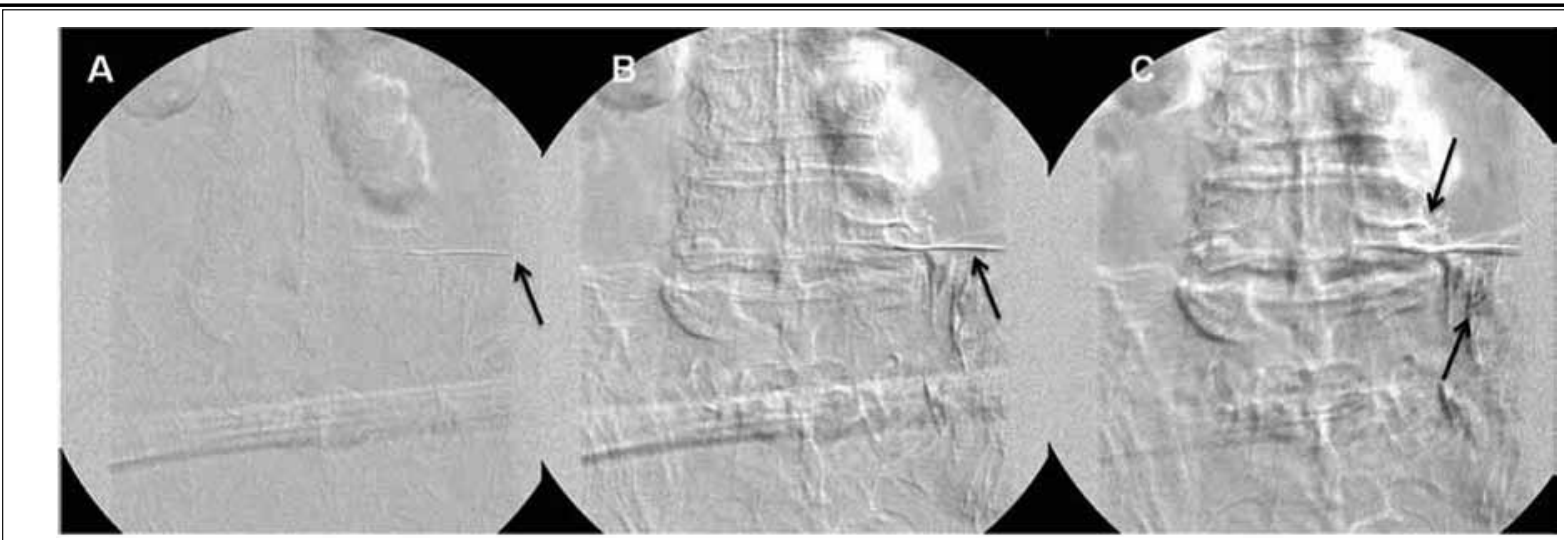

Fig. 5. From Chang Chien et al (4) A. Needle (arrow) entering right L5-S1 transforaminal space; anterior posterior view. B. Injection Phase: Arrow point to spread of contrast medium. No definitive arterial injection observed.

and it is inappropriate to extrapolate that DSA is superior to either static or live fluoroscopy based on Jasper's 3 cases.

Finally, one case report detailed the use of DSA during lumbosacral TFESI (4). An 80-year-old man with severe lumbar spinal stenosis who had previously undergone 2 uneventful lumbar ILESI returned for a right-sided L5-S1 TFESI. Foraminal placement of a 5-inch, 22-gauge Quincke-type spinal needle with a curved tip was confirmed with anterio-posterior (AP), oblique, and lateral views on fluoroscopy. No blood or cerebrospinal fluid (CSF) was seen on aspiration. DSA was performed twice without evidence of intravascular contrast spread (Fig. 5). A test dose of $1 \%$ lidocaine was used without adverse neurological consequences. He was then injected with a $1 \%$ lidocaine and triamcinolone acetonide (insoluble steroid) mixture. The patient immediately reported bilateral lower extremity pain followed by diaphoresis, numbness, and weakness from his lower abdomen downwards. Magnetic resonance imaging (MRI) confirmed an initial T7-T8 spinal cord infarct with later imaging showing the infarct extending from T6-T10. Despite DSA use, arterial uptake of injectate occurred with a subsequent thoracic spinal cord infarct.

\section{Retrospective and Prospective Studies}

McLean et al (11), in a retrospective chart review of 134 patients, compared real-time fluoroscopy versus DSA in 177 cervical TFESI. Intravascular injection was detected in $18 \%$ of cervical TFESIs with real-time fluoroscopy vs. $32.8 \%$ when DSA was used $(P=0.0471)$. Notably, all of the vascular angiograms identified by both live fluoroscopy and by DSA lumbosacral were noted to be venous in nature. Although DSA discovered more venous vascular uptake, it did not discover any more arterial uptake than did fluoroscopy. Since injury and infarction follow arterial and not venous injection of insoluble steroids, one could argue that the use of DSA was superfluous.

Lee et al (9) performed a prospective study of vascular flow detection rate in lumbosacral TFESIs with 60 lumbar and 27 S1 TFESIs. Cases were excluded if the needle placement was deemed difficult or required multiple attempts. The authors found 20 cases of intravascular injection, 9 lumbar and 11 sacral, utilizing DSA. Lumbar TFESIs were done under fluoroscopy using oblique views. Injections were done with a 22-gauge needle inserted inferior to the pars interarticularis into the neuroforamen. The needles were advanced into the "safe triangle" inferior to the pedicle and superolateral to the spinal nerve under biplanar fluoroscopic visualization. For the S1 level TFESIs a 22-gauge needle was inserted into the superior lateral quadrant also using biplanar fluoroscopy. After needle placement each placement was assessed for flash and/or aspiration to look for blood. Contrast injection was undertaken under fluoroscopic as well as DSA imaging and images were saved. If vascular spread was seen, repositioning was attempted. Real-time fluoroscopic guidance with contrast medium predicted 12/20 intravascular injections $(60 \%)$. DSA discovered 8 intravascular injections not identified under fluoroscopy. However, the authors did not distinguish between arterial versus venous uptake. In fact, the authors concede that "the majority of these vascular injections were venous" and not the 
more dangerous arterial injections. A closer look at this study notes that the $\mathrm{S} 1$ injections were associated with a significantly higher proportion $(40.7 \%)$ of vascular uptake when compared to lumbar injections (15\%). This fact is anatomically expected due to the rich sacral venous plexus (20).

El Abd et al (10) evaluated the rate of detection of vascular uptake with DSA that was not identified with other safety precautions including live fluoroscopy, looking for blood flash, aspiration, and a test dose of lidocaine. They enrolled 150 consecutive patients performing 222 TFESIs in the cervical (18.47\%), lumbar $(50.9 \%)$, and sacral levels $(30.36 \%)$. Injections were performed with a 25-gauge 2.5 inch Quincke needle with curved tip for cervical injections; and a 22-gauge 3- or 5-inch Quincke for lumbar and sacral injections. For cervical injections needles were placed under oblique view abutting the superior articular process with forward advancement into the inferior posterior foramen. Lumbar injection needles were placed at the 6 o'clock position under the pedicle with confirmation using an $A P$ view on fluoroscopy. For sacral injections a lateral to medial approach was used with AP view on fluoroscopy and with the needle entering the sacral foramina in the superior lateral position. Prior to DSA employment, aspiration was assessed for a flash at the hub following initial placement, and if aspiration was negative for blood, a test dose injection of $1 \%$ lidocaine was injected, which was considered positive if patients experienced any alteration in sensation, motor weakness, or unusual metallic taste. If these safety measures were negative, DSA imaging was performed to evaluate for vascular needle placement or injectate uptake. If vascular contrast uptake was visualized on fluoroscopy, the needle was repositioned until no further vascular flow was seen prior to DSA imaging. Flash at the hub was seen in 13 injections, aspiration was positive in 11, and live fluoroscopy with contrast detected 46 intravascular flow patterns. DSA identified an additional 5 vascular angiograms not identified on live fluoroscopy. All 5 discovered under DSA and not with live fluoroscopy were venous and were found during sacral injections. This represented $2.25 \%$ of all injections performed in this study.

Kim et al (15) recently performed a large-scale prospective study investigation on the rate of intravascular injections comparing fluoroscopy versus DSA for TFESI. They evaluated 732 injections performed on 348 patients. Injections were performed with a 22-gauge Quincke needle. Lumbar injections were done under oblique fluoroscopic guidance and the needle was inserted inferior to the pars interarticularis. Sacral injections used a cephalad view with imaging of the posterior sacral foramen and the needle was inserted into the superior lateral quadrant. They then aspirated for blood and assessed for a flash at the needle hub. Needle placement was confirmed with biplanar fluoroscopy. Subsequently contrast was injected under live fluoroscopy before performing DSA imaging. Fluoroscopy identified $8.1 \%$ (59 cases) and DSA found $10.5 \%$ (77 cases), including 3.9\% (22 cases) and 6\% (34 cases) at the lumbar level, respectively. Fluoroscopy found $22.6 \%$ ( 37 cases) and DSA found $26.2 \%$ (43 cases) at the sacral level. They found no statistical difference in the identification of intravascular injections between the 2 techniques. Further limitations to this study include the lower number of cases and subsequent dearth of statistical power. Increased venous injection was noted, identified via fluoroscopy and DSA, in older patients and during sacral injections compared to lumbar TFESI, which is consistent with prior findings from other studies mentioned above. Again, no distinction was made by the authors regarding arterial needle placement or arterial medication uptake.

In a similar study, Hong et al (21) evaluated the use of real time fluoroscopy and DSA in lumbar TFESI. This study had 239 patients enrolled and evaluated 249 TFESI between L1 and S1 spinal levels. Thirty-one of 249 TFESI, $8.0 \%$, had vascular uptake as identified by real time fluoroscopy and DSA. Hong et al state 9 of these were identified by DSA alone. Upon further reading of the study, the authors state that in 4 cases "a partial field of intravascular injection was detected" with real time fluoroscopy. This leaves 5 cases in which DSA identified vascular uptake that was not identified with live fluoroscopy. Again, there was no mention by the authors if these additional 5 cases of vascular uptake were arterial or venous.

\section{Meta-Analysis}

A recent meta-analysis by Visnjevec et al (22) compared 4 prospective studies identified by PUBMED, EMBASE, and MEDLINE. The authors analyzed data of 1,290 TFESIs performed with real time fluoroscopy and DSA. One hundred and eighty-eight events were detected by either DSA and/or real time fluoroscopy, 40 of those by DSA alone. The study supports the use and role DSA over real time fluoroscopy by reporting 40 false positives for real time fluoroscopy and 0 false negatives and false positives for DSA (odds ratio 1.32, $P=0.002$ ). 
However, as mentioned above in our own evaluation of those 4 studies $(9,10,15,22)$, there is a failure to further identify if DSA distinguishes arteries from veins. This meta-analysis did not include randomized trials of DSA vs. real time fluoroscopy. As well, all of the procedures investigated were TFESIs, which limits any ability to extrapolate the statistical significance to interlaminar or caudal ESIs.

The advertised advantage of DSA is its potential to identify vascular uptake of injectate. However, as stated above and supported by the above mentioned studies, DSA rarely and unreliably differentiates arterial from venous vascular uptake. In studies in which DSA uptake shows statistical significance in detecting vascular uptake as compared to fluoroscopy, no differentiation was made between arterial and venous uptake or vascular needle placement. Additionally, one study (10) identified a high proportion of DSA positive results seen with sacral TFESI which is expected due to the large venous plexus of the sacral spine. This data is summarized in Table 1. These results have led others (12) to question the ability of DSA to discern arterial versus venous anatomy.

DSA has emerged as a potential additional supportive measure in preventing vascular trespass and has demonstrated greater accuracy in detecting intravascular injections compared to aspiration and live fluoroscopy. DSA is commonly used as an adjunctive safety measure in high-risk procedures such as cervical TFESI and may be useful to clarify an abnormal or unexpected flow pattern observed on live fluoroscopy. The retrospective, prospective, and meta-analysis studies involved in this narrative review had the following results: in a study of 134 patients, McLean et al (11) compared real-time fluoroscopy versus DSA during 177 cervical TFESIs and intravascular injection was detected in $18 \%$ of procedures with real-time fluoroscopy vs. $32.8 \%$ when DSA was used $(P=0.0471)$. Notably, all of the vascular angiograms identified by both live fluoroscopy and by DSA were venous in origin. In one prospective study of vascular flow detection rate in lumbosacral TFESI, Lee et al (9) performed 60 lumbar and $20 \mathrm{~S} 1 \mathrm{TFESI}$ and found 20 cases of intravascular injection, 9 lumbar and 11 sacral, utilizing DSA. Real-time fluoroscopic guidance with contrast medium predicted 12 of the 20 instances. The authors in this study did not distinguish between arterial and venous uptake and conceded that "the majority of these vascular injections were venous" with a statistically significant higher vascular injection rate with S1 TFESI (9). Similar results by El Abd et al (10) were reported in addition to attempting to define the rate of detection of vascular uptake observed by DSA that is missed with traditional safety precautions including live fluoroscopy. One hundred and fifty consecutive patients were enrolled and 222 TFESI were performed in the cervical, lumbar, and sacral spine. Live fluoroscopy with contrast detected 46 intravascular flow patterns with DSA detecting an additional 5 vascular angiograms. However, all 5 of these were venous, and were found while performing sacral TFESI. The results of the Lee et al (9) and El Abd et al (10) studies are consistent with what is known about the rich venous plexus in the sacral epidural space. In the final study of our DSA review, Hong et al (21) illustrates in a head to head comparison of 249 L1-S1 TFESI that DSA did not distinguish the difference between arterial and venous uptake. Additionally, all but 5 of the 31 cases with vascular uptake were discovered with real time fluoroscopy. A recent meta-analysis (22) supports this as well, stating in their own conclusion that of the 4 studies "two studies also noted difficulty discerning arterial versus venous contrast spread for vascular events, but the other 2 did not attempt to make this distinction." These studies suggest that while DSA may detect inadvertent venous injection at a higher rate than live fluoroscopy, there is less robust evidence that DSA enhances recognition of arterial flow and thus may not be useful for preventing the most catastrophic adverse events associated with TFESI.

\section{Discussion}

When reviewing the literature regarding the use of DSA, the concern of this paper was to evaluate if DSA in interventional pain neuraxial procedures can identify arteries from veins. The current narrative review discusses several cases (Table 1) that show that DSA does not provide an ability to distinguish arterial from venous uptake. Additionally, when DSA does show statistical significance in superiority to live fluoroscopy, those studies are limited by high vascular uptake during sacral TFESI, which anatomically is most likely due to increased venous, not arterial, uptake of the injectate. This is specifically important when discussing and comparing arterial and venous adverse reactions from TFESI or ILESI. It is currently a false assumption to conclude DSA has such a capability. More studies need to be performed to specifically distinguish venous from arterial uptake as it relates to DSA use during ESI.

Additionally, severe events are generally difficult to predict when performing axial spine injections whether 
using DSA or not. Caution should be used when contrast patterns are different from expected. The possibility that one is injecting into unintended anatomical vasculature (arterial or venous) structures should warn the interventionalist to reassess and reposition the needle or catheter (8). Safety precautions during epidural injections include appropriate needle placement according to commonly accepted guidelines, negative aspiration, use of extension tubing, a lidocaine test dose following appropriate contrast media, and flow pattern identification under live fluoroscopy. These can all potentially decrease the risk of arterial and venous adverse events even prior to use of DSA as discussed above. These should act as a "red flag" or "stop sign" to not proceed with steroid injection. Although occasionally severe, the sequelae of intra-arterial injection of a short-acting local anesthetic are far more tolerable in most cases than arterial uptake or needle penetration causing a stroke, quadra- or paraplegia, or blindness. Even after negative results with the above mentioned safety precautions, one should consider sole use of soluble steroids as the studies mentioned above call into question the superiority of insoluble steroids. Many authors have suggested that the introduction of insoluble steroid medication into a radiculomedullary artery is the primary cause of spinal cord infarction. Depot steroids such as methylprednisolone and triamcinolone aggregate into clusters of particles ranging from one to greater than $100 \mu \mathrm{m}$, and have the potential to occlude the medullary arterioles measuring $10-15$ $\mu \mathrm{m}$ in diameter (23-24). While interventionalists have long maintained that insoluble steroid medications are superior to soluble counterparts, research to support this belief is less clear (25-26). Some authors have even shown that steroids may not be necessary and have no added benefit over local anesthetics or even saline alone (27-29).

Neuraxial delivery of corticosteroid or local anesthetic is generally well tolerated with a low incidence of serious complications. Even so, adverse outcomes of seizure, irreversible paraplegia, stroke, blindness, and death have been reported from these interventions. DSA has been touted as a radiologic adjunct to interventional neuraxial procedures where it is imperative to identify vascular compromise during the injection. Ensuring that vascular trespass has not occurred serves 2 primary functions. First, it prevents intra-arterial uptake of injectate into the central nervous system.
Second, it insures local deposition of medication and decreased likelihood of a false negative block. Whereas ischemic infarction, respiratory depression, and seizures are attributed to medication delivery to the spinal cord or brain, extra-spinal and venous deposition of medication have far less serious consequences.

DSA identifies vascular uptake which is helpful to many when performing axial spine injections. However, it has not significantly proven its ability to differentiate if the vascular uptake is arterial or venous. It is also important to consider that DSA will not identify, nor prevent, other serious complications such as intra-cord injection or hematoma formation. DSA is limited by motion artifact and images subject to human interpretation. Any motion between the initial scout film and subsequent images will be detected as a change, impairing the subtraction process and degrading image quality $(7,8)$. Thus, utilization of this technology does not negate the potential for human error. The false negative rate of live fluoroscopy is unknown and DSA may provide greater sensitivity and specificity but the exact limits of detection are unclear and the safety profile has not been fully characterized nor validated in research studies.

\section{Conclusion}

It is our opinion that routine use of DSA in interventional pain medicine can distinguish vascular uptake but lacks the consistent ability to differentiate between arterial and venous uptake based on the current scientific and medical evidence. We additionally need to consider that DSA exposes patients, practitioners, and ancillary staff to additional radiation and the current literature fails to demonstrate that it provides additional detection of arterial blood flow beyond that of traditional fluoroscopy. Our recommendation is that further studies need be conducted to investigate if DSA can accurately and consistently identify arteries from veins in axial spine procedure. Interventional pain physicians who use DSA should also employ other safety measures (30) when performing neuraxial procedures (i.e., lidocaine test dose, withdrawing the needle with an unexpected contrast pattern, use of non-particulate soluble steroids, etc.) to decrease the likelihood of an adverse event.

\section{Acknowledgements}

We would like to thank Allison Kessler, MD, for her contributions to the development of this manuscript. 


\section{References}

1. Cohen SP, Bicket MC, Jamison D, Wilkinson I, Rathmell JP. Epidural steroids, a comprehensive, evidencebased review. Regional Anesthesia and Pain Medicine 2013; 38:175-197.

2. Cook TM, Counsell D, Wildsmith JA; Royal College of Anaesthetists Third National Audit Project. Major complications of central neuraxial block: Report on the Third National Audit Project of the Royal College of Anaesthetists. Br J Anaesth 2009; 102:179-190.

3. Smuck M, Fuller BJ, Chiodo A, Benny $B$, Singaracharlu $B$, Tong $H$, Ho S. Accuracy of intermittent fluoroscopy to detect intravascular injection during transforaminal epidural injections. Spine 2008; 33:E2O5-E210.

4. Chang Chien GC, Candido KD, Knezevic NN. Digital subtraction angiography does not reliably prevent paraplegia associated with lumbar transforaminal epidural steroid injection. Pain Physician 2012; 15:515-523.

5. Atluri S, Glaser SE, Shah RV, Sudarshan G. Needle position analysis in cases of paralysis from transforaminal epidurals: Consider alternative approaches to traditional technique. Pain Physician 2013; 16:321-334.

6. Kennedy DJ, Dreyfuss P, Aprill CN, Bogduk N. Paraplegia following imageguided transforaminal lumbar spine epidural steroid injection: Two case reports. Pain Medicine 2009; 10:1389-1394.

7. Rickets JM, Seutter WM. Digital subtraction angiography: Principles and potential applications for emergency medicine. Annals of Emergency Medicine 1985; 14: 41-44.

8. Jasper JF. Role of digital subtraction fluoroscopic imaging in detecting intravascular injections. Pain Physician 2003; 6:369-372.

9. Lee $M H$, Yang KS, Kim YH, Jung HD, Lim SJ, Moon DE. Accuracy of live fluoroscopy to detect intravascular injection during lumbar transforaminal epidural injections. Korean J Pain 2010; 23:18-23.

10. El Abd O, Amadera JE, Pimentel DC, Pimentel TS. Intravascular flow detection during transforaminal epidural injections: A prospective assessment. Pain Physician 2014; 17:21-27.

11. McLean JP, Sigler JD, Plastaras CT, Garvan CW, Rittenberg JD. The rate of detection of intravascular injection in cervical transforaminal epidural steroid injections with and without digital subtraction angiography. PMR 2009; 1:636-642.

12. Davidson J, Robin G. Epidural injections in the lumbosciatic syndrome. $\mathrm{Br}$ J Anaesth 1961; 33:595-598.

13. Buenaventura R, Datta S, Abdi S, Smith $H$. Systematic review of therapeutic lumbar transforaminal epidural steroid injections. Pain Physician 2009; 12:233-251.

14. American Society of Regional Anestehsia. Newsletter. August 2104. http:// asra.com/Newsletters/aug-14.pdf p. 9-10.

15. Kim YH, Park HJ, Moon DE. Rates of lumbosacral transforaminal injections interpreted as intravascular: Fluoroscopy alone or with digital subtraction. Anaesthesia 2013; 68:1120-1123.

16. Baker R, Dreyfuss P, Mercer S, Bogduk $N$. Cervical transforaminal injection of corticosteroids into a radicular artery: $\mathrm{A}$ possible mechanism for spinal cord injury. Pain 2003; 103:211-215.

17. Verrills P, Nowesenitz G, Barnard A. Penetration of a cervical radicular artery during a transforaminal epidural injection. Pain Medicine 2010; 11:229-231.

18. Landers MH. Comments on: Penetration of a cervical radicular artery during a transforaminal epidural injection. Pain Medicine 2010; 11:1306-1307.

19. Rathmell JP, Michna E, Fitzgibbon D, Stephens LS, Domino KB. Injury and liability associated with cervical procedures for chronic pain. Anesthesiology 2011; 114:918-926.

20. Lin VW, Bono CM, Cardenas DD, Frost FS, Hammond MC, Lindblom LB, Perkash I, Stiens SA, Woolsey RM. Spinal Cord Medicine Principles and Practice. Second Edition. Dermos Medical Publishing, NY, NY, pp 3-21.

21. Hong JH, Huh B, Shin HH. Comparison between digital subtraction angiography and real-time fluoroscopy to detect intravascular injection during lumbar transforaminal epidural injections. Reg Anesth Pain Med 2014; 39:329-332.

22. Visnjevec $\mathrm{O}$, Kim $\mathrm{P}$, Farid-Davari $\mathrm{S}$, Johnson P, Nader ND. Digital subtraction angiography versus real-time fluoroscopy for detection of intravascular penetration prior to epidural steroid injections: Meta-analysis of prospective studies. Pain Physician 2015; 18:29-36.
23. Benzon HT, Chew T-L, McCarthy RJ, Benzon HA, Walega DR. Comparison of the particle sizes of different steroids and the effect of dilution: A review of the relative neurotoxicities of the steroids. Anesthesiology 2007; 106:331-338.

24. Tiso RL, Cutler T, Catania JA, Whalen $K$. Adverse central nervous system sequelae after selective transforaminal block: The role of corticosteroids. Spine J 2004; 4:468-474.

25. Kennedy DJ, Plastaras C, Casey E, Visco C), Rittenberg JD, Conrad B, Sigler J, Dreyfuss P. Comparative effectiveness of lumbar transforaminal epidural steroid injections with particulate versus nonparticulate corticosteroids for lumbar radicular pain due to intervertebral disc herniation: A pospective, randomized, double-blind trial. Pain Med 2014; 15:548-555.

26. El-Yahchouchi C, Geske JR, Carter RE, Diehn FE, Wald JT, Murthy NS, Kaufmann TJ, Thielen KR, Morris JM, Amrami KK, Maus TP. The noninferiority of the nonparticulate steroid dexamethasone vs the particulate steroids betamethasone and triamcinolone in lumbar transforaminal epidural steroid injections. Pain Med 2013; 14:1650-1657.

27. Friedly JL, Comstock BA, Turner JA, Heagerty PJ, Deyo RA, Sullivan SD, Bauer Z, Bresnahan BW, Avins AL, Nedeljkovic SS, Nerenz DR, Standaert C, Kessler L, Akuthota V, Annaswamy T, Chen A, Diehn F, Firtch W, Gerges FJ, Gilligan C, Goldberg H, Kennedy DJ, Mandel S, Tyburski M, Sanders W, Sibell D, Smuck M, Wasan A, Won L, Jarvik JG. A randomized trial of epidural glucocorticoid injections for spinal stenosis. N Eng J Med 2014; 371: 1-21.

28. Manchikanti L, Falco FJ, Hirsch JA. Lumbar interlaminar epidural injections are superior to caudal epidural injections in managing lumbar central canal stenosis. Pain Physician 2014; 17:E691-E702.

29. Bicket MC, Gupta A, Brown CH, Cohen SP. Epidural injections for spinal pain: A systematic review and meta-analysis evaluating the "control" injections in randomized controlled trials. Anesthesiology 2013; 119:907-931.

30. Rathmell JP, Benzon HT, Dreyfuss $P$, Huntoon M, Wallace M, Baker R, Riew D, Rosenquist RW, Aprill C, Rost NS, Buvanendran A, Kreiner DS, Bogduk $\mathrm{N}$, Fourney $\mathrm{DR}$, Fraifeld $\mathrm{E}$, Horn $\mathrm{S}$, 
Pain Physician: May/June 2016: 19:255-266

Stone J, Vorenkamp K, Lawler G, Summers J, Kloth D, O'Brien Jr. D, Tutton S. Safegaurds to prevent neurologic complications after epidural steroid injections: Consensus opinions from a multidisciplinary working group and national organization. Anesthesiology 2015; 122:974-984. 